\title{
Relativistic Numerical Simulations of Superluminal Sources
}

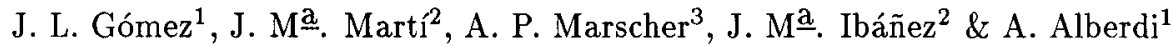

Abstract. We study the generation and evolution of superluminal components in relativistic jets through relativistic hydrodynamical and emission simulations of a square-wave perturbation in the jet velocity.

\section{Generation and Evolution of Superluminal Components}

In our study of the physics of relativistic jets the fluid dynamics are computed using a time-dependent $2 \mathrm{D}$ relativistic hydrodynamic code developed by Martí et al. (1997; and references therein). Superluminal components are generated by introducing a temporary square-wave increase in the beam Lorentz factor from $\Gamma=4$ to $\Gamma=10$. The resulting dynamical evolution of the perturbation along an initially overpressured jet model is shown in Fig. 1. The first panel corresponds to the quiescent jet, where the pattern of recollimations and rarefactions caused by the initial overpressure of the jet is evident. The perturbation quickly evolves into a shock, trailed by a rarefaction. While the shock moves with a constant pattern speed of about $\Gamma=10$, the shocked material speed fluctuates substantially as the shock passes through compressions and rarefactions in the underlying jet. When the perturbation passes through a standing shock, the latter is "dragged" downstream for some distance before returning to its initial position as the steady jet becomes reestablished. Perturbations on the beam surface saturate in oblique shocks within the beam trailing the main perturbation (see also Gómez et al. 1997). The evolution of the perturbation seems to proceed without noticeable variations in the number of particles contained within it, maintaining approximate self-similarity, and being consistent with an adiabatic evolution.

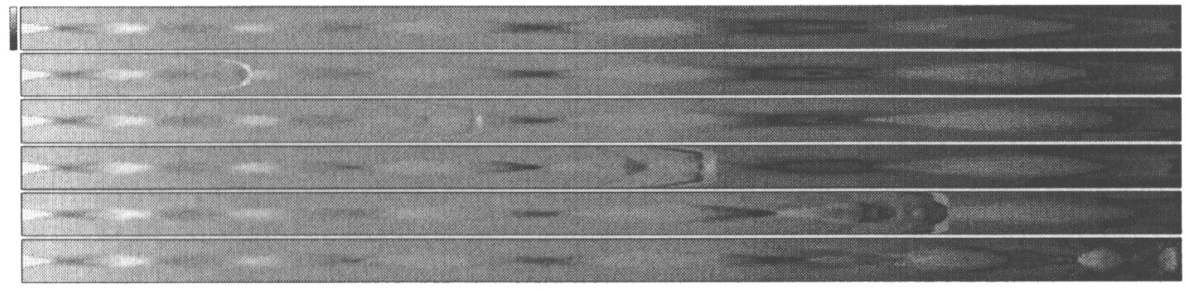

Figure 1. Pressure distribution at six epochs after the introduction of a squarewave perturbation to the flow Lorentz factor for the jet model discussed in the text. Owing to the decreasing pressure in the atmosphere, the jet expands with a mean opening half-angle of $\sim 0.4^{\circ}$.

\footnotetext{
${ }^{1}$ Instituto de Astrofísica de Andalucía (CSIC), Granada, Spain

${ }^{2}$ Departamento de Astronomía y Astrofísica, Universidad de Valencia, Valencia, Spain

${ }^{3}$ Department of Astronomy, Boston University, Boston, USA
} 

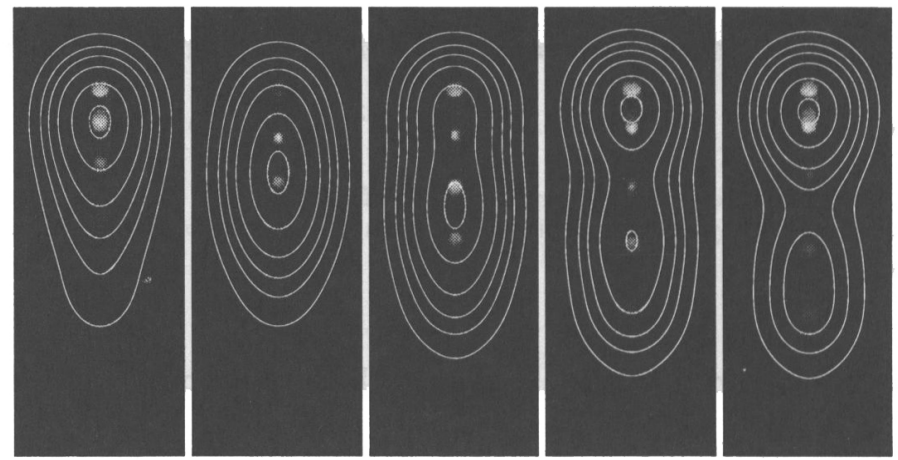

Figure 2. Total intensity radio maps for the stationary and four subsequent epochs (from left to right) for the jet model of Fig. 1. Maps correspond to optically thin observing frequency, and $10^{\circ}$ viewing angle. Contours show the emission convolved with a circular Gaussian beam. The jet inlet is at the top of each panel.

\section{Radio Emission}

Full Stokes parameters determining the radio emission from the jet model whose hydrodynamics are computed as before are calculated by integrating the synchrotron transfer equations accounting for all the special relativistic effects, including time delays within the jet (see Gómez et al. 1997, and references therein, for more details). In the convolved maps in Fig. 2, we recognize the usual corejet VLBI structure of a blazar, with a single well-defined traveling component associated with the moving shock. We obtain an apparent velocity of $\sim 8.6 c$, in general concordance with the expected value for a pattern Lorentz factor of $\Gamma \sim 10$ and viewing angle of $10^{\circ}$. The unconvolved maps show a much more complex jet structure, consisting of a regular pattern of knots of high emission associated with the internal oblique shocks. Due to the time delays, the shocked region appears as a very extended region of higher emission, which is moving and interacting with the quiescent jet. As a result of this interaction, the previously stationary hotspots in the jet increase in flux, being later dragged temporarily downstream. Significant indications of this dragging effect have been observed in 3C 454.3 (Marscher et al. 1997), where the core of the source seems to be associated with the first recollimation shock, and is temporarily dragged downstream by the ejection of a new component. Similar results of the interaction of superluminal and stationary components have also been observed in $0735+178,3 \mathrm{C} 279$, Centaurus A (Tingay et al., these proceedings, p. 87), and may be expected in other sources as more high-frequency images become available.

Acknowledgments. This research is supported in part by the Spanish DGICYT (PB941275, PB94-0973) and by NATO grant SA.5-2-05(CRG.961 228). J. L. Gómez and J. Ma Martí gratefully acknowledge return grants from the Spanish Ministry of Education.

\section{References}

Gómez, J. L., et al. 1997. $A p J, 482, \mathrm{~L} 33-36$.

Marscher, A. P., et al. 1997. BAAS, 29, 847.

Martí, J. Mà. et al. 1997. $A p J, 479,151-163$. 